\title{
Emprendimiento femenino en los grados de Maestra de la Facultad de Ciencias de la Educación de la USC
}

\section{Female Entrepreneurship in the degrees of teachers of theFaculty of Educational Sciences of the University of Santiago de Compostela}

\author{
Milena Villar, Aixa Permuy, María del Mar Sanjuán \\ Universidad de Santiago de Compostela, España
}

\begin{abstract}
Resumen
El objetivo del póster que se presenta es visibilizar aquellos factores que influyen en el emprendimiento femenino de las mujeres universitarias que cursan los grados de maestra en educación infantil y primaria en la promoción 2014-2015. El interés se centra fundamentalmente en indagar en los factores económicos, sociales, emocionales y formativos que afectan a estas mujeres, y en base a ellos elaborar una propuesta de formación que los equilibre y mejore. Para ello se empleó un método de investigación descriptivo y un diseño transversal. La muestra del estudio, estuvo constituida por 46 personas. El instrumento utilizado en la investigación, de corte cuantitativo, es una escala tipo lickert conformada por un conjunto de ítems presentados en forma de afirmaciones. Los resultados mostraron que las variables relativas a la economía y a la asunción de riesgos son las que condicionan en mayor medida el camino hacia el emprendimiento de estas mujeres.
\end{abstract}

Palabras clave: emprendimiento, mujeres, factores económicos.

\begin{abstract}
The objective of the poster is visible factors that influence female entrepreneurship among women graduates in grades teacher in early education and promotion 20142015. The interest was mainly focused on investigating in economic, social, emotional and educational factors affecting these women, and based on them develop a training proposal that balances and improves. For this a descriptive research method and a crossover design was used. The study sample intentionally character, consisted of 46 students. The instrument used in research has been instrumental quantitative measure, namely a lickert type scale consists of a set of items presented as statements. The results showed that the variables related to the economy and risk taking are what determine further the road to entrepreneurship of these women.
\end{abstract}

Keywords: entrepreneurship, women, economic factors.

La Universidad de Santiago de Compostela se encuentra a la vanguardia de los centros universitarios gallegos y españoles con la voluntad puesta en encontrar respuestas en el presente a los interrogantes del futuro. Por lo tanto, puede definirse como una Universidad completa, con titulaciones en las cinco grandes áreas del conocimiento y con un Campus de Excelencia Internacional que busca devolver a la sociedad el esfuerzo realizado en $\mathrm{I}+\mathrm{D}+\mathrm{i}$ con progreso, riqueza $\mathrm{y}$ bienestar sostenido en el conocimiento. Este campus compostelano está llamado a convertir Galicia en una referencia académica e investigadora para el resto del mundo

Es de señalar que la USC (s.f.) cuenta con una serie de valores que la caracterizan, como son su tradición y experiencia; su modernidad y proyección de futuro; su vocación y proyección universales; el compromiso con la docencia; la calidad en la docencia, la investigación y los servicios; el vanguardismo en cuanto a los equipos de investigación, así como la innovación y el desarrollo; la conexión con el mundo de la empresa, la investigación aplicada y el desarrollo tecnológico, el compromiso con el deporte, la cultura y la sociedad, y, el compromiso con el emprendimiento.

La población objeto de estudio se enmarca en la Facultad de Ciencias de la Educación, de la propia USC.

En este centro se cursan actualmente los grados de Maestro/a en las especialidades de Educación Infantil y Educación Primaria, el grado en Educación Social y el grado en Pedagogía. También se imparten diversos posgrados, unos de iniciación a la investigación, otros de orientación profesionalizadora y otros mixtos.

Es en las titulaciones universitarias de grado de Maestra/o en educación primaria y educación infantil de la Facultad de Ciencias de la Educación, en concreto en el último curso, donde se ha llevado a cabo el estudio y donde se va a llevar a cabo la formación, debido a las necesidades detectadas por parte de la investigación realizada a una muestra de 46 alumnas a través de la aplicación de un cuestionario elaborado ad hoc.

\section{Estudio de necesidades: método}

En el estudio se ha empleado un método de investigación descriptivo y un diseño transversal.

La muestra del estudio, de carácter intencional, estuvo constituida por 46 alumnas pertenecientes al último curso de los grados en Educación Infantil y Educación Primaria.

El instrumento utilizado en la investigación, de corte cuantitativo, es una escala tipo lickert conformada por un conjunto de ítems presentados en forma de afirmaciones, antes los cuales los sujetos tienen que manifestar su opinión eligiendo uno de los puntos o categorías de la escala. Estas puntuaciones varían entre manifestar un grado de total acuerdo hasta un grado de total desacuerdo o indicar NS/NC. La escala contiene 79 ítems o afirmaciones en escala de intervalo, organizadas al azar para evitar posibles sesgos y relativas a los indicadores económicos, psicológicos y/o emocionales, sociales y formativos. Es de señalar que los ítems se han formulado de manera positiva para una mejor 
comprensión de los mismos. Previamente el instrumento presenta ítems de identificación relativos al sexo, a la edad, a los estudios, una pregunta abierta acerca de su idea de negocio, y un último ítem relativo a la forma jurídica de dicho negocio. Es de señalar que este instrumento ha sido elaborado ad hoc, teniendo en cuenta la población destinataria y ha sido validado a través de un juicio de personas expertas.

\section{Estudio de necesidades: resultados}

Las principales barreras para el emprendimiento femenino en el contexto de la Facultad de Ciencias de la Educación son, principalmente, las relativas a variables económicas tales como la consecución de financiación y fondos de entidades financieras, las ayudas al emprendimiento femenino, la posesión de recursos económicos suficientes y los requisitos para concurrir a las ayudas públicas al emprendimiento; las relativas a la asunción de riesgos, tales como conseguir aumentar las ganancias de su negocio y ofrecer un producto que no existe en la oferta del mercado, y aquellas referidas las variables relacionadas con las redes sociales orientadas a negocios. A pesar de que estas variables condicionan a todas las personas emprendedoras, en este contexto se marcan como las principales barreras de la falta de emprendimiento en mujeres universitarias. Para ello, se elabora una propuesta de formación que satisfaga dichas necesidades y contribuya a crear mujeres seguras, arriesgadas y con los conocimientos necesarios para crear su proyecto empresarial.

\section{Propuesta de acción}

Una vez descritas las necesidades y conocido el contexto donde tendrá lugar la implementación de la formación se presentan los principales elementos que constituyen la misma.

El proyecto que se lleva a cabo se fundamenta en una propuesta de formación que se basa en aspectos económicos, de financiación y ayudas al emprendimiento femenino, así como cuestiones relacionadas con la asunción de riesgos. El nombre que recibe esta propuesta es "Econorriesgo, emprendiendo como mujer, arriesgando como jugadora”.

Tras una ardua investigación acerca de las principales barreras que dificultan el emprendimiento de las mujeres universitarias de las titulaciones de la Facultad de Ciencias de Educación de la Universidad de Santiago de Compostela se han obtenido una serie de resultados que indican que estas se refieren principalmente a barreras relativas a variables económicas tales como la consecución de financiación y fondos de entidades de financiación, las ayudas al emprendimiento femenino, la posesión de recursos económicos suficientes y los requisitos para concurrir a las ayudas públicas al emprendimiento, relativas a la asunción de riesgos, tales como conseguir aumentar las ganancias de su negocio y ofrecer un producto que no existe en la oferta del mercado, referentes al asesoramiento burocrático y relacionadas con las redes sociales. A pesar de que estas condicionan a todas las personas emprendedoras, en este contexto marcan las principales barreras de la falta de emprendimiento en mujeres universitarias. En base a esto, se ha considerado necesario elaborar una propuesta de formación que trate de paliar dichas barreras.
Un importante desafío al que se enfrentan todas las iniciativas emprendedoras, en cualquier parte del mundo, es el acceso a capital para iniciar un negocio. De todos/as es sabido que el dinero proporciona tranquilidad y el respaldo que se necesita, sobre todo en los inicios; abre puertas para conseguir otras financiaciones; facilita el contacto con otras personas que también han emprendido; permite invertir tiempo y recursos para afinar al máximo la idea de negocio y buscar oportunidades rentables; entre otros beneficios. Emprender requiere, cada vez más, partir de unos recursos que aseguren una inversión inicial adecuada y un margen temporal suficiente para hacer viable la iniciativa y poder vivir de ella. Pero el acceso a esos recursos económicos, ya sea en forma de financiación o ayudas, es más difícil para las mujeres que para los hombres (Facundo, 2011).

Aunque originalmente se creyó que los negocios gestionados por mujeres recibían un trato menos favorable por parte de los proveedores de fondos que aquellos pertenecientes a varones, actualmente existen evidencias claras de que no existe discriminación en las tasas de aprobación o denegación de deuda, sino que las divergencias obedecen a diferencias sistemáticas en las características de las empresas propiedad de hombres y mujeres (Orseret al., 2006, cit. en Rodeiro y Fernández, 2011).

Ya sea debido al sexo o a las características de las empresas de las mujeres lo que está claro es que los aspectos económicos y el miedo al riesgo suponen un problema para ellas en el momento de emprender.

No se puede afirmar que exista igualdad a la hora de emprender, en primer lugar porque las emprendedoras parten de unos niveles de renta menores que los emprendedores, aspecto el cual tiene consecuencias importantes en el modelo de negocio que se desarrollará, ya que a menor capacidad de inversión, menor dimensión del negocio, menor creación de empleo, menor internacionalización y menor implicación en sectores económicos que requieren fuertes inversiones iniciales (Facundo 2011). Esta desigualdad salarial es uno de los factores que provoca, en parte, la dificultad de las mujeres para emprender debido a la falta de financiación.

A pesar de esta menor riqueza personal, la cual supone una menor acumulación de capital financiero para invertir en sus negocios inferior al de sus colegas masculinos, es de señalar que, de media, ellas siempre han aportado a sus negocios igual o más dinero que los emprendedores y siguen invirtiendo de su bolsillo más de lo que lo hacen los hombres (Facundo, 2011).

Otro aspecto a destacar es que las mujeres poseen una menor experiencia profesional y en dirección de empresas, según explican Rodeiro y Fernández (2011). Esto puede dificultar la relación con los proveedores externos de fondos a la hora de cerrar tratos, entre otros motivos porque tampoco estén acostumbradas a trabajar con entidades financieras (Brush et al., 2000, cit. en Rodeiro y Fernández, 2011). Este hecho puede intimidarlas y desanimarlas a la hora de buscar financiación externa; según Cavalluzzoet al. (2012, cit. en Rodeiro y Fernández, 2011) las emprendedoras 
solicitan menos créditos porque creen que no serán capaces de conseguirlos, no tienen confianza en ello.

Además, las mujeres no persiguen un crecimiento en sus negocios o prefieren dirigir empresas que experimentan un crecimiento lento (Orser y HogartScott, 2003; Jennings y Cash, 2006, cit. en Rodeiro y Fernández, 2011) y como consecuencia no buscan financiación externa. Las empresas que no persiguen el crecimiento de una forma explícita o que no aspiran a internacionalizarse, pueden resultar poco atractivas para la concesión de financiación por parte de las entidades bancarias (Neergaardet al., 2006, cit. en Rodeiro y Fernández, 2011).

Así, las entidades financieras pueden percibir aquellos sectores donde las mujeres tradicionalmente establecen sus actividades como menos rentables, puesto que se trata de sectores convencionales donde los márgenes de beneficios son más reducidos (Orseret al., 2006, cit. en Rodeiro y Fernández, 2011). Asimismo, cuando inician sus actividades en sectores tradicionalmente masculinos, donde las entidades financieras no perciben un riesgo diferencial, las mujeres pueden ser consideradas unas deudoras más arriesgadas en la medida en que o bien suelen tener menos experiencia, o bien consideran que es insuficiente para competir en el sector (Neergaardet al., 2006, cit. en Rodeiro y Fernández, 2011). Segundo Facundo (2011), las entidades de crédito y otras fuentes de inversión sí discriminan en base al tamaño de la empresa, el sector de actividad o su potencial de crecimiento. Por mucho que las emprendedoras ajusten bien las inversiones, las entidades financieras no van a apoyar sus negocios si no está claro el rendimiento y, sobre todo, el retorno del crédito. En cualquier caso, se trata en un endurecimiento de las condiciones de los proveedores/as de fondos al prestar sus recursos a unos negocios que consideran más arriesgados.

Por otra parte, las empresas dirigidas por hombres tienden a buscar financiación externa en mayor medida, a través de redes sociales que facilitan al proveedor conocer mejor al emprendedor y su negocio; en cambio, las mujeres prefieren fuentes de financiación internas, se centran en redes sociales cuyos inversores informales son compañeras, amigos cercanos y empleados en los cuales puede confiar. Siguiendo a Facundo (2011), es de señalar que existe resistencia a solicitar fondos entre las propias mujeres emprendedoras. Estas suelen preferir fuentes cercanas a su realidad, es decir amistades y familiares, en lugar de fuentes más externas como los instrumentos financieros, los préstamos bancarios o las inversiones de capital riesgo. Justo lo contrario de lo que ocurre con los emprendedores, quienes son más propensos a recurrir a este tipo de fuentes externa de financiación, y, por lo tanto, dispuestos a asumir más riesgos.

En lo referente al riesgo, las mujeres emprendedoras mantienen una actitud más precavida con sus inversiones iniciales. Estas mujeres no quieren hacer crecer su empresa, porque quieren mantener el control sobre esta y no depender de terceras personas o instituciones, o incluso porque ya prevén que recibirán un trato diferente y no desean exponerse a una solicitud de financiación denegada.
Este hecho de asumir menos riesgos, dificulta en mayor medida su carrera profesional, ya que como asumen menos riesgos piden menos dinero, y como piden menos dinero al final no hacen todo lo que quieren y no llegan tan lejos. Tienen que aprender a pedir más (Ana María Llopis, 2009, cit. en Facundo, 2011).

A pesar de todo lo explicado, es necesario comprender que hay aspectos que no dependen de las propias decisiones o características personales y que limitan las posibilidades de inversión inicial. Son factores estructurales que individualmente no se pueden modificar y que se deben tomar en cuenta porque juegan un papel importante en la dimensión económica del negocio $\mathrm{y}$, en la confianza crediticia que este genera (Facundo, 2011).

Por lo tanto, esta propuesta de formación se va a centrar en aquellos factores que sí pueden cambiarse, en aquellos aspectos personales y profesionales que necesitan de una mejora para superar las barreras económicas y seguir adelante.

Los objetivos que se pretenden alcanzar con esta propuesta formativa son, a nivel general, superar las barreras económicas y los miedos que llevan a las mujeres a no emprender, y a nivel específico, conseguir financiación y/o fondos de entidades de financiación, conocer las ayudas existentes al emprendimiento femenino, reunir los requisitos necesarios para concurrir a las ayudas públicas al emprendimiento, presentar candidatura a las ayudas existentes, asumir riesgos, emprender sin miedo a lo que pasará, poseer una mayor autoconfianza, fomentar la capacidad emprendedora en las mujeres y dominar las redes sociales orientadas al empleo.

Es de señalar que toda la formación tendrá lugar en la Facultad de Ciencias de la Educación, en el Campus Norte, por ser la ubicación más cercana para las asistentes.

Previamente al comienzo de la propuesta de formación, se llevará a cabo una primera sesión, con las mujeres universitarias. Este primer contacto servirá de base para que las mujeres destinatarias conozcan al personal formador, $y$ este les pueda enseñar $y$ proporcionar información y técnicas adecuadas partiendo de los conocimientos previos de las mimas. Para esto, las formadoras/es llevarán a cabo la técnica de brainstorming, de forma que las universitarias comuniquen lo primero que se les ocurra sobre una determinada cuestión relacionada con la formación. Además, en esta primera sesión tendrá lugar una entrevista grupal, para conocer con más detalle las necesidades de las futuras emprendedoras. Por otra parte, esta primera sesión tiene la finalidad de asignar un mentor o mentora a cada una de las participantes para que se encargue de su mentorizada a lo largo de todo el proceso de formación, enseñándole, aconsejándola, guiándola y ayudándola en su desarrollo invirtiendo tiempo y conocimientos. Esta sesión servirá también para determinar con las personas asistentes, un horario que permita conciliar los distintos tiempos de cada mujer. 
Los módulos que conforman esta propuesta formativa son los siguientes:

Módulo I: Líneas de financiación pública y privada y nuevas posibilidades de financiación. Este módulo se centrará en dar a conocer a las mujeres emprendedoras las principales líneas de financiación pública y privada, dado que muchas tienen dificultades para acceder a la financiación. Muchos de los problemas son debidos a que la información de las ayudas públicas está muy dispersa y es difícil de localizar, por lo que en este módulo se pretende agruparlas, dividiéndolas en públicas y privadas. Además, pretende ofrecer a las participantes alternativas de financiación a las ya existentes, dada la dificultad de obtener financiación para desarrollar el proyecto de empresa deseado. Este módulo tendrá una duración de 2 horas divididas de la siguiente forma:

- Conceptos básicos sobre financiación (1/2 hora). En esta sesión se tratarán temas básicos sobre financiación, tales como en que consiste el proceso de financiamiento, los tipos de financiación a corto y largo plazo, y las fuentes de financiación que conocen. Estos temas se tratarán a través de una tormenta de ideas en la cual cada mujer deberá aportar sus conocimientos sobre el tema. Posteriormente se llevará a cabo una puesta en común y una breve exposición sobre el tema.

- Líneas de financiación (1 hora). Se iniciará esta sesión con un debate acerca de las líneas de financiación que cada una conoce. Tras el mismo, se llevará a cabo una exposición sobre dichas líneas, proporcionando a las asistentes mediante el aula virtual, material sobre las mismas. Además, se les invitará a presentar su candidatura a estas ayudas, y se les explicará el procedimiento que deben seguir para su solicitud. Por último, se llevará a cabo un caso práctico de manera individual para que decidan, según el caso, que tipo de financiación es el más adecuado.

- Alternativas disponibles a la financiación tradicional (1/2 hora). Se dará comienzo a esta sesión con una entrevista grupal acerca del conocimiento que poseen las participantes sobre alternativas de financiación para su futura empresa. Posteriormente se les explicará la existencia de estas nuevas posibilidades de financiación (familia y amistades, "business angels”, capital riesgo y fuentes especiales, entre otras).

Módulo II: Ayudas al emprendimiento femenino. Este módulo supone un pilar de especial importancia en esta formación, al tratarse de ayudas específicamente destinadas al colectivo femenino. Tiene una duración de 1 hora distribuidas de la siguiente manera:

- Ayudas económicas al emprendimiento femenino. En esta sesión se les dará a conocer a las participantes las diversas ayudas que existen al emprendimiento femenino en España, y en concreto, el programa Emega de apoyo al emprendimiento femenino.

Módulo III: La constante del riesgo al emprender. Dado que las mujeres son menos propensas a arriesgarse a la hora de emprender que sus compañeros varones, este módulo pretender ofrecer al colectivo femenino una visión más positiva del riesgo, enseñándoles a minimizarlo y enfrentarse a él. Tiene una duración de 4 horas distribuidas de la siguiente forma:
- Enfrentándose al riesgo (1 hora). En primer lugar se analizará con las mujeres la mayor aversión al riesgo, como colectivo, en contraposición a sus homólogos masculinos y se iniciará un debate acerca de las causas que provocan esto. Posteriormente, se les enseñará a perder el miedo a arriesgarse, haciendo que reconozcan el riesgo como algo natural que está presente en todos los ámbitos de la vida través de diversas actividades. Además, se les explicará la importancia de planificar para perder parte de ese miedo y elaborar presupuestos cerrados.

- Modelo de éxito (1 hora presencial y 2 virtuales). En la segunda parte de la sesión tendrá lugar una exposición por parte de una emprendedora de éxito, la cual les explicará a las participantes los riesgos que ha tenido que asumir y los miedos que ha debido afrontar para alcanzar su meta. Posteriormente, cada mujer deberá intervenir exponiendo un caso concreto en el que haya tenido que afrontar una situación de riesgo, o en su caso, afrontar un miedo, de manera que se den cuenta de que si quieren pueden hacer lo que se propongan. Se les solicitará que visionen la película de Coco Avant Chanel (Fontaine, 2009), y la comenten en un foro centrándose en la variable riesgo.

Módulo IV: El plan de negocio como instrumento de análisis de la viabilidad y búsqueda de financiación. Este módulo pretende servir de base para que el colectivo femenino sea capaz de elaborar un plan de empresa viable y con altas posibilidades de financiación. Tendrá una duración de 8 horas divididas de la siguiente forma:

- Fases de elaboración de un plan de negocio (2 horas presenciales y 2 virtuales). En este momento se les explicará a las asistentes a la formación como se elabora un plan de empresa adecuado y viable. Se les asignará un caso por grupos de 4 personas cada uno, y deberán elaborar un plan de negocio en base a los conocimientos adquiridos. Se podrá entregar al final de la formación. Se abrirá un foro virtual para solventar las posibles dudas que puedan surgir.

- Importancia del plan económico-financiero (1 horas presencial y 2 virtuales). Se iniciará la sesión con un debate acerca de la importancia del plan económicofinanciero. Posteriormente se podrán en común los aspectos más relevantes y se expondrá la importancia del mismo. Se les proporcionará a las asistentes un manual de creación de un plan de empresa y un manual para la realización de un plan financiero. Se les solicitará que lean en profundidad estos documentos, y se abrirá otro foro para responder a posibles dudas.

Módulo V: Redes sociales orientadas a negocios y empleo. Este módulo tiene como objetivo formar a las mujeres asistentes en aspectos relacionados con las redes sociales orientadas a los negocios. Se pretende que las conozcan, que sepan de su existencia, que sean capaces de crear perfiles en las mismas y aprovecharlas al máximo para su beneficio profesional. Tiene una duración de una hora:

- Diferentes tipos de redes sociales (1 hora). Se les mostrará la existencia de diferentes redes sociales relativas al empleo más allá de la conocida Linkedin, se 
les animará a crear un perfil y se les proporcionará información básica sobre su funcionamiento.

Como se puede observar a lo largo de todo el proceso de formación, la metodología presenta un carácter magistral combinada con actividades de carácter más práctico. Además, se denota un aprendizaje basado en la comunicación, el trabajo en equipo y el learning by doing. Es de señalar, como ya se ha especificado a lo largo de la propuesta, que la formación tendrá un carácter presencial en algunas ocasiones y virtual en otras, en función del tipo de actividad a realizar, y abarcará una duración de 15 horas (9 de ellas presenciales).

En cuanto a los medios necesarios, debemos diferenciar entre recursos materiales, didácticos, humanos y económicos. Los primeros harán referencia a ordenadores, cañón de proyección, pantalla de visualización, papelógrafo, encerado, mesas y sillas y espacios para la formación y para los talleres. En cuanto a los recursos didácticos necesarios, estos serían un aula virtual, material de escritura, manuales sobre financiación, documentos sobre ayudas al emprendimiento femenino, manual sobre la elaboración de un plan de empresa y película basada en la asunción de riesgos. Los recursos humanos esenciales en esta propuesta son un/a tutor o tutora, personal formador experto, mentoras y mentores y un/a profesional gestor/a.

Esta propuesta de formación estará financiada por la Universidad de Santiago de Compostela.

Como toda formación, está propuesta será evaluada integralmente, llevando a cabo los siguientes tipos de evaluación, con sus correspondientes instrumentos.

- Evaluación inicial: Se lleva a cabo a través de la aplicación de un cuestionario elaborado para tal fin y del análisis de los resultados obtenidos. Esto servirá de base para partir de las necesidades y de los conocimientos previos, en caso de disponer de los mismos, de las mujeres destinatarias de la propuesta de formación y conocer sus estructuras mentales para así poder modificarlas o completarlas.

- Evaluación continua: También se realizará una evaluación procesual durante el período que dure el proceso formativo. Algunos indicadores a tener en cuenta serían la implicación en la temática propuesta, el interés por realizar las actividades, su dinamismo y motivación, entre otras. Así, se podrán conocer y determinar los avances del colectivo destinatario mediante la observación. Además, se evaluará la implementación de la propuesta llevando a cabo para esto reuniones individuales y grupales, que permitan recoger información acerca de la aplicación de la propuesta formativa.
- Evaluación final: Para determinar si se cumplieron los objetivos iniciales se aplicará una escala de satisfacción, para conocer su grado de satisfacción en relación con la formación recibida, y descubrir aspectos susceptibles de mejora; un cuestionario dicotómico, para conocer el nivel de adquisición de competencias en materia de recursos económicos, financiación y asunción de riesgos por parte de las personas destinatarias, y un supuesto práctico que deberán resolver aplicando las competencias adquiridas.

- Evaluación de impacto: Para determinar el impacto de esta formación en el colectivo destinatario se llevará a cabo un seguimiento del mismo, utilizando para ello reuniones periódicas y entrevistas individuales una vez finalizado el proceso de formación. Así, de esta manera, se podrá conocer la generalización de los contenidos de la propuesta de formación a la realidad.

- Evaluación de proceso: Durante la implementación de la propuesta de formación se registrarán datos sobre la puesta en práctica de las acciones formativas (tiempos de ejecución, materiales utilizados, temporalización, etc.) en el diario de sesiones del/a formador/a que contribuirán a reformular, si fuese necesario, aquellos aspectos que así lo requieran en posteriores ediciones de la formación.

Por último, una vez finalizada la propuesta, el personal formador $\mathrm{y}$ organizador de la misma elaborarán una memoria final, en la cual se recogerá toda la información de la evaluación llevada a cabo, para posteriormente ser enviada a los servicios de emprendimiento universitario de la Universidad de Santiago de Compostela.

\section{Referencias}

Facundo, G. (2011). El libro rojo de las mujeres emprendedoras. Barcelona: Libros de cabecera.

Fontaine, A. (dir.) (2009). Coco avantChanel. (Película). Francia: Haut et Court / Warner Bros.

Rodeiro, D. y Fernández, S. (2011). El emprendimiento femenino en el sistema universitario español y gallego. Santiago de Compostela: USC.

Uniemprende (2013). Manual de creación de un plan de Empresa y manual para la creación de un plan financiero. Materiales en pdf del curso on-line de financiación de empresas de base tecnológica. Santiago: Autor.

Universidade de Santiago de Compostela (s.f.). Historia de la USC. Disponible en:

http://www.usc.es/es/info_xeral/historia/

USC. (2014). Plan Estratégico. Santiago: Autor. Disponible en: http://www.usc.es/export/sites/default/gl/goberno/vrcali dade/descargas/proplanestrUSC_2011-20.pdf 